CP, 2014 , Nº4, pp. 87-100. ISSN 2014-6752. Girona (Catalunya). FERNÁNDEZ, Paola: Consumos culturales en América Latina y la emergencia del prosumidor: Un recorrido conceptual desde la Sociedad de la Información. Recibido: 05/05/2014 - Aceptado: 17/07/2014

\title{
CONSUMOS CULTURALES EN AMÉRICA LATINA Y LA EMERGENCIA DEL PROSUMIDOR: UN RECORRIDO CONCEPTUAL DESDE LA SOCIE- DAD DE LA INFORMACIÓN.
}

\section{Cultural consumption in Latin America and the emergence of the prosumer: a concep- tual journey from the Information Society.}

\author{
Autora: FERNÁNDEZ, Paola \\ Licenciada - Universidad Nacional de Quilmes - Argentina -fernandezp28@gmail.com
}

\section{Resumen}

Los procesos de digitalización imponen nuevos criterios de jerarquización, promoción, distribución y acceso a la creación y el conocimiento. Los cambios en los patrones de uso cotidiano, la transformación en los circuitos de producción de contenidos, entre otros aspectos, habilitaron la emergencia de nuevos actores, como es el caso del prosumidor. En este escenario, las prácticas a nivel usuario presentan diferencias sustanciales, de acuerdo a los contextos socio-culturales de los cuales emerjan, así como los niveles de acceso a las tecnologías.

El presente trabajo se orienta a (re) pensar las prácticas sociales en el consumo de contenidos, a partir de la irrupción de nuevos dispositivos tecnológicos. Los objetivos se orientan a explorar y analizar el escenario mediático actual -desde las prácticas sociales y no sólo desde el dispositivo tecnológico- en el marco de la Sociedad de la Información, con el propósito de sistematizar los alcances y limitaciones en el accionar a nivel usuario en la región latinoamericana; dado que es una región que se ve interpelada, sobre todo, por las corrientes de estudio norteamericanas y que precisa de un enfoque local.

Desde esta perspectiva, el artículo intenta ser un aporte a los estudios culturales de comunicación en América Latina, que en la actualidad enfrentan al desafío de identificar al nuevo mapa de relaciones de los actores, dentro del nuevo ecosistema comunicacional convergente.

En base a la revisión de la bibliografía disponible, el artículo describe el ecosistema mediático actual, inserto en la Sociedad de la Información y como altera los consumos culturales en la región.

\section{Palabras clave}

Consumos Culturales, Prosumidor, Sociedad de la Información, producción, participación.

\begin{abstract}
The digitization impose new criteria for ranking, promotion, distribution and access to creative and knowledge. Changes in the patterns of daily use, processing circuits in content production, among others, fitted out the emergence of new actors, such as the prosumer. In this scenario, the user-level practices differ substantially, according to the socio-cultural contexts in which they emerge, and the levels of access to technology.

This paper aims to (re) thinking social practices in content consumption, from the emergence of new technological devices. The objectives are aimed at exploring and analyzing the current media landscape-from the social practices and not only from the device technology-in the context of the Information Society, in order to systematize the scope and limitations in the actions at user level in Latin America; Since it is a region that is challenged, especially by American study streams and requires a local approach.

From this perspective, the article is intended as a contribution to cultural studies of communication in Latin America, which currently face the challenge of identifying the new map of relations between actors within the new converged communications ecosystem.

From the review of available literature, the article describes the current media ecosystem, insert in the Information Society and alters as cultural consumption in the region.
\end{abstract}

\section{Key words}

Cultural consumption, Prosumer, Information Society, production, participation. 


\section{Introducción}

Los cambios en la lógica de circulación y reproducción de la información atraviesan un momento disruptivo en la actualidad (Vercelli, 2009). En consecuencia, los estudios de comunicación se enfrentan al desafío de identificar al nuevo mapa de relaciones de los actores, dentro del nuevo ecosistema comunicacional convergente. Desde esta perspectiva, la cultura de masas y el modelo análogo de comunicación son considerados obsoletos, frente al cual emerge el proceso denominado por Manuel Castells como "autocomunicación de masas" (2010: 88), que adquiere el potencial de alcanzar una audiencia de carácter global, distribuida al tiempo que es el usuario quien produce y selecciona el mensaje a compartir.

En este contexto, los usuarios migran de los medios tradicionales hacia medios, digitales, interactivos y en línea, especialmente aquellos que facilitan el acceso a redes sociales y a contenidos audiovisuales en un marco delineado por Jesús Martín Barbero (2010) por la convergencia digital

Como aclaración metodológica, es pertinente considerar la multiplicidad de pantallas y canales de difusión, que requieren de contenidos y aplicaciones atractivas (Levis, 2011), por lo cual es de mayor precisión la denominación de "pantallas monofunción"l o "multifunción". Se trata de comprender que la diferencia entre los medios y las pantallas actuales radica en la funcionalidad de cada una -de acuerdo a la circunstancia-, sus características ergonómicas, apropiaciones y significaciones que cada sujeto realice de ellas. Así se sostiene que la distancia entre los llamados "nuevos" y "viejos" medios o pantallas no es tal, en virtud de apreciar su función orgánica como dispositivo de mediación (Fernández, 2013). Es decir, se mantiene la genealogía de la pantalla, al tiempo que se transforma su funcionalidad orgánica. A partir de esta premisa, podemos encontrar una validación a lo expuesto en el uso de los teléfonos móviles, utilizados en la actualidad también para el visionado de contenidos audiovisuales.

Este recorrido es pertinente dada la convergencia entre pantallas a la hora de plantear el circuito de los contenidos -escritos y audiovisuales- y acciones de los usuarios, ya que son estos últimos quienes, en definitiva, alteran las lógicas de producción y circulación a través de sus prácticas. En otras palabras, las prácticas sociales alteran el uso original con el que fue concebido el dispositivo pero no la genealogía de la pantalla. Es la herramienta tecnológica la que se adapta a las necesidades del individuo y altera sus funciones originales, en un proceso de co-construcción continuo (Vercelli, $\left.2009^{3}\right)$.

\section{Objetivos}

El objetivo principal del presente trabajo se encuentra en describir los nuevos Consumos culturales en América Latina y sistematizar el nuevo actor emergente, denominado prosumidor en el territorio latinoamericano.

Las razones por las cuales se delimita este campo de análisis se fundamenta en contribuir y enriquecer el conocimiento de los estudios culturales en América
Latina. Este punto se encuentra vinculado a que la investigación de los nuevos consumos culturales es robusta en Europa y Estados Unidos pero difiere en los consumos culturales propios de la región latinoamericana. De esta manera, se intentará sistematizar los aspectos que identifican a los usuarios en la región e identificar sus nuevos hábitos de recepción.

\section{Metodología}

La estrategia metodológica de esta investigación es de carácter descriptivo y exploratorio. Para los objetivos planteados, se ha realizado un relevo de bibliografía específica, a través de fuentes teóricas especializadas, relacionadas con la capacidad del usuario-final en el uso de Tecnologías de la Información y la Comunicación -en adelante, TIC- para producir y consumir información digital. 
A partir de esta línea de acción, el enfoque metodológico se orientó al relevo de estudios latinoamericanos vinculados a los términos prosumidor, producción y consumos culturales, Sociedad de la Información y Tecnologías de la Información y la Comunicación (en adelante, TIC). La trascendencia del tema se fundamenta en interpretar el nuevo contexto mediático, a fin de comprender las acciones emergentes de los usuarios en el marco de las Industrias Culturales.

Como elemento transversal al análisis, se completó el estudio con la investigación desarrollada por el Observatorio Iberoamericano de la Ficción Televisiva: (2011; 2012), con el propósito de recaudar información específica de los mecanismos de emisión y hábitos de recepción de contenidos televisivos

\section{Ecosistema mediático 2.0: actores, acciones y coyuntura de emergencia}

Los procesos de digitalización emergentes en 1980 (Negroponte, 1999) y la explosión de la denominada World Word Web -Red de redes o Internet-, crearon las condiciones de posibilidad para la aparición de nuevos recursos narrativos y textuales, imposibles de imaginar en el mundo analógico.

De esta observación, se identifican las configuraciones actuales del mercado audiovisual, que converge entre dos tendencias en apariencia, contrapuestas: por un lado, las nuevas tecnologías que abaratan costos de producción y distribución (Zallo, 2007), que amplían las ventanas de comercialización, disponibilidad de los contenidos y la posibilidad para que los consumidores puedan archivar, interactuar, apropiarse y hacer recircular las producciones mediáticas.

En paralelo, el escenario mediático se encuentra atravesado por la concentración de la propiedad - de los medios comerciales dominantes-, con oligopolios y monopolios multinacionales que controlan todos los sectores de la industria del entretenimiento (McChesney, 2002). En este sentido, se adhiere a lo observado por Martín Becerra (2010), cuando explica que:

"el sector de las tecnologías constituye el centro de la gravitación de la re-estructuración económica del capitalismo avanzado. La progresión de los cambios suscitados mediante dicha re-estructuración -y que se producen dentro de la misma estructura social-, irá encauzándose en el proyecto de la Sociedad de la Información" (pp: 87).

\subsection{Sociedad de la Información y participación}

A partir de este enfoque, se comprende a la Sociedad de la Información como una reducción drástica del costo y tiempo necesarios para almacenar, procesar y transmitir información. En consecuencia, estos cambios en las relaciones de precios afectan la organización de la producción, distribución de bienes y servicios y las relaciones laborales de producción (Zallo, 2007). De este último factor, se desprende la formulación complementaria que aporta la perspectiva de las políticas de comunicación, que entiende a la Sociedad de la Información como el conjunto de herramientas orientadas a dotar de dirección y coherencia a la diseminación de recursos info-comunicacionales, en virtud de su centralidad productiva (Becerra, 2010).

En este contexto, es posible comprender a la Sociedad de la Información como marco en el que se ubica el nuevo ecosistema mediático en el que conviven productores, usuarios, tecnologías, propietarios y consumidores, sin olvidar organismos supranacionales -como la Organización Mundial de Comercio (OMC); la Organización Mundial de Propiedad Intelectual (OMPI); la Unión Internacional de Telecomunicaciones (UIT), entre las de mayor influencia a nivel mundial en lo referente al campo de las tecnologías y los negocios en torno a ellas- y Estados nacionales.

Sin embargo, la región latinoamericana se encuentra atravesada por desigualdades estructurales. En sentido estricto, corresponde distinguir entre los diferentes niveles de acceso en función de las apropiaciones de los ciudadanos de las TIC y las desigualdades entre países; denominada como brecha digital, definida como la división en el acceso físico que impide el crecimiento y desarrollo económico, tecnológico y social. En relación, cerca de un cuarto o incluso un 
tercio de la población no tiene acceso a computadoras e Internet (Van Dijk 2005 en Becerra, 2010:97).

En consecuencia, los usuarios se relacionan a partir de diferencias de acceso y participación, por lo cual las tecnologías e Internet emergen como condiciones de posibilidad para alterar los vínculos entre los actores que intervienen en los procesos de producción. Según advierte Luis García Fanlo (2012):

Los usuarios juegan nuevos roles dentro del sistema mediático, relacionado con la funcionalidad que le otorguen a la pantalla. Desde esta perspectiva, el individuo realiza acciones y prácticas que, sin la multifuncionalidad permitida por las nuevas pantallas, eran inimaginables. De esta última afirmación, es preciso analizar la coyuntura a fin de aportar al campo de conocimiento de la convergencia entre televisión, telefonía celular y plataformas Web, en particular las redes sociales, aparecen como condiciones de posibilidad para que la conjunción entre soporte tecnológico y prácticas sociales sirva como espacio privilegiado para que se desarrollen y potencien (...) las luchas que se caracterizan por la imposición unilateral de los productores sobre los consumidores. (p: 110).

En palabras de Roberto Igarza (2008), la participación del usuario en Internet deja entrever el perfil de un consumidor que alterna con más facilidad que antes entre el rol pasivo y un rol más activo como productor, como fuente de información.

Desde este abordaje, es posible partir del plano de la recepción para identificar nuevos indicadores de participación en el ecosistema mediático.

\subsection{Prosumidor y participación: un emergente de las prácticas de recepción}

Se comprende la participación como la posibilidad de que el público -ya sean grupos o individuos- genere contenidos y disponga de espacios donde emitirlos, dados los tiempos excluyentes de los medios análogos, propios de la estructura de los contenidos pensados para el formato televisivo. En esta tensión de enfoques y fuerzas de producción, la participación de los usuarios transforma el escenario audiovisual y habilita la emergencia de un nuevo actor, denominado prosumidor. El término es un acrónimo de los conceptos de "productor" y "consumidor", que sostiene la producción de información o contenidos propios por parte de los usuarios. El concepto fue anticipado por Marshall McLuhan y Barrington Nevitt (1972), quienes afirmaron que la tecnología electrónica permitiría al consumidor asumir simultáneamente los roles de productor y consumidor de contenidos. McLuhan (1997) infería que en la edad pos-eléctrica los actores comunicativos resentirían profundas transformaciones resultantes de la complejidad inherente a los nuevos ambientes comunicativos. La noción de prosumidor advierte particular relevancia en la "ecología de medios" (Islas, 2008:8). A partir de esta perspectiva, Howard Rheingold (1997) anticipó el valor de las comunidades virtuales, anclado en la experiencia y en los individuos que (re)descubren el poder de la cooperación, comprendida como un juego, un modo de vida: una fusión de capital de conocimiento, capital social y comunión - entendida como el ritual de socialidad en la red-. En relación, es posible inferir que el rol del prosumidor abarca acciones, interacciones, intercambios, transacciones comerciales, vínculos sociales, entre otros. A partir de este diagnóstico, el circuito de producción actual se encuentra atravesado por nuevas prácticas comunicativas, sujetas en la experiencia y en la participación de los individuos, en un circuito que se retroalimenta en carácter continuo; perceptibles en el ecosistema mediático.

\section{Contexto en América Latina: un análisis del prosumidor desde el territorio}

El nuevo contexto comunicacional que introducen las comunicaciones digitales móviles, estimulan la creatividad y la autonomía. Así, la colaboración es una práctica comunicativa que distingue a los prosumidores en los nuevos ambientes comunicativos que desplaza consigo el desarrollo de las redes digitales e Internet (Islas, 2014).
Como ejemplo de los nuevos hábitos que introducen las TIC, el teléfono móvil ha registrado importantes transformaciones hasta convertirse en indispensable y multifuncional dispositivo de comunicaciones. En el imaginario de la sociedad de la ubicuidad ${ }_{4}$ el rol del avanzado dispositivo admite ser considerado como fundamental. El teléfono móvil ha trascendido a la condición de "terminal tonta" y hoy permite tener 
acceso a Internet; recibir y contestar correos electrónicos; asegurar el acceso a servicios de televisión móvil, mapas y avanzados servicios de localización; ser utilizado como cámara fotográfica, grabadora y avanzada remediación del popular "walkman"; asumir las funciones propias del mando a distancia, e inclusive desempeñarse como útil dispositivo de almacenamiento de información digital (Islas, 2014).

Sin embargo, el consumo de Internet desde dispositivos móviles es del 51\% en Latinoamérica: muy por debajo de África y el Medio Oriente, que es del 93\% e incluso de Europa, con el 58\% y Asia con el 48\% (GSMA, 2013 $)$. Este punto es importante si se considera que las acciones de los prosumidores incluye la participación en los medios de comunicación desde múltiples dispositivos (García Fanlo, 2012; Jenkins, 2008; Islas, 2008, 2014; Scolari, 2009).

En este sentido, en la región, los precios, la infraestructura y el servicio de Internet móvil en América Latina impiden un desarrollo pleno del sector info-comunicacional, muy concentrado en pocas manos (Telefónica en Argentina, el grupo Slim/Telmex en México, por ejemplo ${ }_{6}$ )

Pese a las particularidades de la infraestructura, María Vasallo de Lopes (2012) reconoce que las audiencias y los usuarios se manifiestan activamente: son selectivos, autodirigidos y productores tanto como receptores de textos. También son plurales y múltiples, aunque diversos, fragmentados e individualizados. Así, categorías clave como las de elección, selección, gusto, fans, intertextualidad, interactividad son transversales al nuevo escenario mediático.

La era digital es un espacio de intercambio permanente de significados desde una lógica concreta, que es la participación. La plataforma que ofrecen los sitios Web ha hecho posible que el usuario, sobre todo los jóvenes, establezcan el principio de la "manifestación" como soporte para la vida en la red, entendida como las expresiones volcadas en Internet (Teniente, 2004:3)

A partir de estas líneas, podemos identificar los grados de participación realizados por las audiencias a fin de caracterizar los cambios emergentes. Si bien la categoría de prosumidor refiere en lo específico a la gestión y producción de la información y contenidos por parte de los usuarios (Jenkins, 2009), podemos desmembrar una primera aproximación en el plano de la recepción. Así, a través de la incidencia en el proceso comunicacional a partir de la interacción ${ }_{7}$, interactividad y (re) circulación de textos por parte de los usuarios, se comprenden las acciones de los sujetos como elementos disparadores para identificar los escenarios mediáticos nacionales y regionales. Los prosumidores son efectivos actores en la gestión del conocimiento y el consumo cultural (Islas, 2008), por lo cual es necesario enmarcar sus acciones dentro de la Sociedad de la Información y los bienes comunes a fin de que el término no se limite a la difusión de contenidos audiovisuales o de entretenimiento. No debemos cegarnos con las ilusiones que proporcionan las tecnologías de la información y la comunicación en cuanto a la democratización de acceso y participación, ya que se corre el riesgo de no reconocer el poder de concentración de los actores privados, como la corporación Google por ejemplo (Vercelli, 2009).

En línea, Denis de Moraes (2010) destaca que el uso de las tecnologías puede favorecer mayores contactos, interacciones e intercambios, incluso introducir nuevos formatos, lenguajes y dinámicas en los procesos comunicacionales, siempre y cuando se extienda el campo de análisis para reconocer las nuevas estrategias de acumulación de capital simbólico y económico a través de la cultura y la comunicación, para identificar que las principales fuentes emisoras se encuentran concentradas en manos de corporaciones transnacionales centradas en Estados Unidos, Europa y Japón (De Moraes, 2010), razón necesaria para ahondar en todo el conjunto de factores que inciden en el proceso de producción y difusión de los productos culturales. Resulta fundamental evaluar quien controla la variedad de la oferta, cuál es su naturaleza ideológico-cultural, cuáles son los contenidos y las prioridades establecidas en las programaciones. Por lo tanto, no basta con alardear acerca del incremento de opciones sin verificar sus intenciones y énfasis, así como sus efectos socioculturales e influencia en la formación de la opinión pública. (De Moraes, 2010:72). 


\subsection{Ficción y participación: una aproximación al rol del prosumidor}

Es posible retomar los principios elaborados por el Observatorio Iberoamericano de la Ficción Televisiva (Obitel, 2011; 2012), con el objetivo de sistematizar las prácticas de los usuarios y establecer sus cánones de acción desde el plano de la ficción. Este anclaje diferencia los consumos culturales en la región latinoamericana de otros territorios -como Estados Unidos o Europa-, por sus oposiciones en el marco del consumo de bienes culturales para evitar generalizar las prácticas y hábitos de visionado televisivo, consecuencia inevitable dada la penetración cultural en América Latina de productos de procedencia estadounidense ${ }_{8}$

A partir de esta caracterización, se sistematiza el grado de participación de las audiencias en relación a las posibilidades que crean los dispositivos tecnológicos. En esta línea de análisis, las redes sociales se convierten en ejemplos reales que permiten medir, analizar e interpretar las interacciones de las audiencias, a la vez que habilita construir un número estimativo de "conversación en tiempo real", En este sentido, se construyen como comunidades y grupos formados por intereses comunes, estimulados por la socialidad virtual, además de favorecer la circulación ampliada y el intercambio de materiales audiovisuales.

En el plano de la ficción y la participación, las experiencias de recepción caracterizan los grados y tipo de prácticas de comunicación tanto por parte de las emisoras como de las audiencias, en virtud de la participación del televidente/usuario a través del uso de múltiples plataformas -páginas Web, blogs, redes sociales, plataformas colaborativas como YouTube, entre otros-. La experiencia de recepción y participación referida en este punto depende de la oferta de navegación que habilite la productora del contenido audiovisual y del vínculo a retroalimentar con su audiencia.

Sin embargo y desde América Latina, pensar en términos de recepción, no representa novedades para los estudios culturales de la región, ya que puede trazarse un paralelismo con el concepto de multimediaciones propuesto por Guillermo Orozco Gómez (1997). La propuesta del autor, a su vez, operacionaliza -a través de categorías específicas- el concepto de mediaciones, argumentada por Jesús Martín Barbero (1987). En este marco de referencia, Orozco Gómez identifica que toda relación esta mediada por tecnologías ${ }_{10}$, ya que las audiencias se encuentran atravesadas por un conjunto de influencias que provienen de la mente del individuo preconceptos, experiencias- y del contexto sociocultural donde esté inmerso.

Así, la interacción entre televisión y audiencia no se reduce al momento de estar frente a la pantalla sino que está condicionado e influenciado antes y después de la emisión audiovisual.

Los espectadores/usuarios encuentran una forma de reproducir los contenidos que mejor se adapten a sus gustos y los jerarquizan de una forma a la que la televisión no puede llegar, incluso por las propiedades de las nuevas pantallas, signadas por la evolución tecnológica e informativa que transformó por completo las posibilidades de intercambio, circulación, consumo y almacenamiento de información así como también la producción simbólica cultural, multimediática y multilingüe. (Orozco Gómez, 2009: 2)

Desde esta perspectiva, Orozco Gómez (1994) complementa la idea de Martín-Barbero (1987) al entender la mediación como un proceso de recepción donde se recrean significados y sentidos, elemento que posibilita su sistematización -a través de categorías de análisis- desde la interacción de la audiencia con el medio y con las otras instancias sociales. Es decir, "las múltiples mediaciones especifican las distintas influencias que inciden en los procesos de interacción entre audiencias y pantallas y colaboran en el resultado de la negociación para que se acepte o se rechace el significado propuesto" (Orozco Gómez, 2009:3)

Inserto en la misma corriente epistemológica, Carlos Scolari (2009), interpreta la convergencia mediática como una conjunción entre distintos lenguajes y medios, donde la interactividad crea las condiciones para un grado de interlocución inédito en relación a los cambios en los modos de producción y consumo. Este punto es consecuencia de la digitalización, que permite la reproducción y la disponibilidad de los contenidos en todo momento y lugar -con la obvia dependencia del servicio de Internet y computadora-. A este nivel de mediación, el autor lo denomina Hipermediaciones, proceso que atrae nuevas capacidades interpretativas y nuevas dinámicas de 
consumo, ya que las tecnologías participativas profundizan el proceso al ofrecer al usuario la posibilidad de crear y distribuir sus propios contenidos, por lo cual es preciso revaluar el rol de las audiencias (Scolari, 2009:289).

En otras palabras, el usuario está influenciado por numerosas relaciones que acarrean los usos de múltiples dispositivos tecnológicos, situación que implica revaluar su relación con los medios análogos pero también con las narrativas y formatos.

En América Latina, el análisis de los consumos de recepción revelan prácticas de comunicación tanto por parte de las emisoras o canales de televisión como por parte de las audiencias, que se caracterizan en función del grado de participación del televidente/usuario a través del uso de múltiples plataformas -redes sociales, blogs, sitios de Internet, entre otros-. Este tipo de participación puede ser observada sea en plataformas y modelos construidos por las propias emisoras o en las redes sociales habilitadas por los usuarios para interaccionar e interactuar con el contenido. En ambos casos, se constatan la apropiación de temas y de tramas de las ficciones televisivas por parte de las audiencias, acciones que relevan grados distintos de implicación (OBITEL, 2012).

En línea, a partir del análisis de los tipos de interacción ${ }_{11}$ con los contenidos en las páginas webs y redes sociales, así como los niveles de interacción que las audiencias desarrollan a partir de los contenidos; es posible inferir que interacción y compromiso son fenómenos heterogéneos cuyas diferencias atraviesan la vida cotidiana y determina los hábitos y patrones de consumo cultural.

En paralelo, estas nuevas prácticas fueron acompañadas por iniciativas desde las productoras televisivas para incorporar las prácticas de fans de las audiencias, tanto en Internet como en las redes sociales; no sólo como estrategia comercial y divulgación de contenidos ficcionales sino también como forma de lograr un compromiso de la audiencia con los productores del programa. De este último punto se desprende una de las principales diferencias con los consumos culturales estadounidenses -e incluso europeos-: no es significativa la producción propia de contenidos ${ }_{12}$, como si lo es en Estados Unidos (Jenkins, 2009).

El uso de nuevas tecnologías favorece la interacción del usuario con los medios de comunicación, no sólo en la participación directa sino con la posibilidad de hacer un producto completo, desde el guión hasta la edición y programación, con oportunidades de visibilizar su creatividad. Y es esta posibilidad lo que sería importante potenciar y desarrollar en las plataformas digitales (Casajus; Guerrero, 2014).

En este contexto, los usuarios están presentes y activos, tal es el caso de las redes sociales (Facebook y Twitter, por mencionar ejemplos). En América Latina, los programadores de contenidos ofrecen desde sus portales Web la posibilidad de participación mediante la posibilidad de publicar comentarios y valorizar contenidos por parte de los usuarios. En este punto, también es importante la actualización constante de la información en a red y generar aplicaciones específicas que permita la participación de los mismos (Ibídem). En este sentido, las redes sociales y blogs también actúan como plataformas para aprovechar la iniciativa de los públicos en el ecosistema digital y contribuir a la difusión de contenidos por ellos generados; sobre todo en consonancia con los usos y hábitos de las nuevas generaciones que tienden a un modelo de consumo basado en información fragmentada (Igarza, 2008).

\section{Prosumidores: alcances y limitaciones de una nueva práctica de consumo}

A partir de lo expuesto respecto a los grados de recepción -como indicadores de la participación de los usuarios en la red-, es posible establecer un marco de situación en lo que alude el término prosumidor.

Desde la perspectiva de la red, prosumidor es aquel usuario que produce y promueve contenidos propios a partir de sus interacciones en la red, y que nutren al ecosistema. Sin embargo, las complejidades son latentes y la problematización de los contenidos sociales necesarias. En palabras de Gabriela Sued (2010), el cambio en la producción de contenidos y en el rol de los usuarios instala la tensión entre la posibilidad de una nueva lógica de producción cultural y la subordinación del usuario, ahora devenido en productor, a la lógica impuesta por los medios masivos de comunicación. 
En línea con el argumento, se puede encontrar en Facebook, a modo de ejemplo, que la participación en la red social, se caracteriza por una producción de contenidos vinculada al consumo de una plataforma.

A partir de estas consideraciones, es posible prever que la producción refiere tanto a información y contenidos como significación social. Incluso, tal como postula Germán Villarreal (2010), es posible considerar el conjunto de acciones y prácticas como intrínseca a la estructura de la red. Así, en el mundo de las redes sociales, hasta las más simples acciones, como establecer o suspender conexiones entre usuarios, contribuyen a la construcción del grafo social que es el sustrato significativo de toda la comunidad y del medio en sí.

De este modo, todos los miembros de una comunidad virtual están interconectados en red y establecen relaciones interpersonales, sean de carácter directo o indirecto. Desde esta aproximación, se infiere que estas prácticas sociales en la red se encuadran dentro de las tantas mediaciones que las atraviesan (Orozco Gómez, 2001), de las cuales las tecnologías son aquellas que median en lo que refiere a todos los aspectos del campo mediático. En el plano de recepción, podemos anclar la relación entre redes sociales y televisión a partir de reconocer las apropiaciones que los usuarios realizan de ellas para compartir sus experiencias con la "pantalla chica", no tanto desde el fanatismo, sino más bien desde la comprensión del funcionamiento de la narrativa televisiva.

En este sentido, ver televisión es hablar, comentar, jugar y todo a la vez. En el escenario actual, el consumo cultural se define por la convergencia entre las pantallas, donde las redes sociales operan como canales de circulación para vehiculizar recomendaciones, quejarse por los cambios de horario, conversar acerca de las historias y los personajes, entre otras prácticas.

El móvil y las redes refuerzan los canales de conversación y potencian la función del diálogo que hizo trascendente la televisión en la vida cotidiana (Amado, 2013). Se desprende de aquí la afirmación de Omar Rincón (2013), que la relación entre relatos y narraciones, constituyen un repertorio desde donde producir significación en la vida cotidiana.

Este punto es clave para configurar el escenario de la televisión actual, a partir de las representaciones sociales que los espectadores perciben en los contenidos audiovisuales, pero también, para marcar las distancias necesarias con producciones y consumos extranjeros. Así, en ambientes comunicativos regionales, el prosumidor o prosumidores, expuesto a un extenso repertorio de recursos multimedia, emprenden determinadas acciones comunicativas, como expresar sentimientos, difundir información, gestionar información, compartir conocimientos, a través de dispositivos e interfaces digitales móviles o estacionarias, para, en un proceso autológico, propiciar las condiciones necesarias de vinculación comunitaria con otro prosumidor o prosumidores que bien pueden formar parte de una red social-. Al explorar las capacidades de los dispositivos e interfaces digitales, los prosumidores gestarán las condiciones necesarias para acceder a ambientes comunicativos más complejos, transitando a una nueva ecología cultural. (Islas, 2008:17)

Las tecnologías y las propuestas que de ellas se derivan representan oportunidades para impulsar el desarrollo de otro tipo de televisión, configurada a partir de las vinculaciones que el usuario realice con los contenidos. Se infiere para este objetivo, la necesidad -desde la producción de contenidos- del desarrollo de nuevas temáticas y narrativas, que interpelen a las audiencias a través de sus gustos, mejoren los recursos narrativos e incorporen relatos atractivos para el público desde representaciones que lo respeten y no lo estereotipen.

Sin embargo, para que lo mencionado pueda ser un escenario real, es necesario reconocer las limitaciones impuestas por la propia conformación de la red, así como también las limitaciones impuestas por los propietarios de los sitios, servidores y portales. De este último punto, se desprende que el usuario no puede compartir libros o películas cuyo derecho de autor no lo permita e incluso, encuentra restricciones legales para actuar de intermediario (Vercelli, 2009).

\subsection{De la práctica a las restricciones}

Desde un plano estructural, el término prosumidor conlleva limitaciones propias del término y de la coyuntura donde emerge.

Para analizar desde un plano integral las prácticas de las audiencias en las redes sociales -tanto en la interacción virtual como en la producción de 
contenidos- es necesario contextualizar lo que las redes permiten realizar a partir de su arquitectura, es decir, la estructura "física" que no puede ser modificada por el usuario, en términos de Lawrence Lessig (1998). Así, desde un primer momento, el accionar del usuario está condicionado por las "paredes" impuestas por la propia red social. Sin embargo, el concepto se encuentra atravesado por las tensiones latentes entre el determinismo tecnológico y el social 13 .

Desde esta perspectiva de análisis, los defensores del primero sostendrán que la producción en Internet, cuyo ejemplo es posible encontrar en Manuel Castells (2012). En consonancia, el autor reconoce el proceso de transformación cada vez más influyente que producen las TIC, al punto de identificar que "la sociabilidad real se da hoy en Internet" (en prensa) ${ }_{14}$. Sin embargo, pese a que la participación de los usuarios en la red es amplia (Obitel, 2012), dista de conformar la interacción plena. No obstante, autores como Henry Jenkins (2008, 2009); Carlos Scolari (2009) y Guillermo Orozco Gómez (2012), coinciden en que este tipo de prácticas de los usuarios interpelan a las empresas productoras de contenidos con un alto grado de libertad.

A partir de esta consideración, se identifica un tipo de acción que altera las prácticas tradicionales de producción y circulación de la información, sobre todo si no limitamos el término a lo que implica la creación de contenidos audiovisuales. Así, desde las categorías de Lawrence Lessig (1998), la propia estructura de la red impide la participación ilimitada de los ciudadanos.

La afirmación se apoya en lo que el autor denomina como una "espada de doble filo" capaz de asegurar la confidencialidad de las comunicaciones de los individuos y monitorear sus acciones cotidianas.

En consecuencia, describe los distintos códigos que permiten o impiden a los usuarios del ciberespacio atravesar diversas prácticas. Y no sólo a través de efectos directos -por modelo, se puede considerar que la plataforma colaborativa YouTube elimine un contenido particular de un usuario-, sino también por efecto indirecto. Por ejemplo, es el caso de un código que permite la acción de usuarios registrados e identificados con una empresa, por lo cual sus comentarios estarán condicionados por miedo a las consecuencias en el "mundo real".

Desde este anclaje metodológico, el escritor de "Las leyes del ciberespacio" (1997), con la intención de plantear un sendero que permita desmitificar la abstracción "irregulable" de Internet, desde él cual se traza el escenario mediático en el cual interaccionan empresas, intermediarios ${ }^{12}$, usuarios y productores.

Para construir las normas regulatorias, el autor fundamenta cuatro categorías que inciden como restricciones en el uso de Internet:

- Ley: acepción de mayor trascendencia e investigación epistemológica, que no son ni más ni menos que el conjunto de normas que los organismos nacionales y supranacionales establecen para un uso “óptimo" de Internet. Desde esta óptica, las leyes impuestas para su regulación son en su mayoría de carácter penalizador que fueron -y son- enfocadas en su mayoría a los usuarios ${ }_{15}$.

- Normas sociales: las que actúan, en palabras de Lessig (1998), como una "segunda restricción reguladora”. Son el conjunto de valores y normas que rigen la vida de los individuos y que condicionan sus prácticas en la red. Puede interpretarse como una auto-regulación cultural, distinta según las costumbres y hábitos de cada región.

- Mercado: al igual que en el mundo real, Internet también se encuentra condicionado por la lógica comercial. Las bases del mercado condicionan la participación de otros actores, sobre todo por la característica intrínseca de las Industrias Culturales ${ }_{16}{ }^{\prime}$ tendientes a la concentración y lo referente a la imposición en los precios de los productos y servicios. En lo que compete a las intenciones del presente artículo, las producciones de sujetos individuales o colectivos por fuera de la lógica comercial, no serán competitivas, ni para la industria ni en su reproducción y circulación -con contadas las excepciones $_{17^{-}}$, lo que dificulta su trascendencia fuera de su zona de influencia - en las redes sociales, sería la denominada como comunidad virtual-. Esta restricción, afirma el especialista en derecho informático, constriñe en el ciberespacio como lo hace en el espacio real. Dicha limitación, interpela en directo lo que significa el precio de pago por servicio de Internet, diferencial y desigual en las diversas partes 
del mundo ${ }_{18}$.

-

Código: en este punto el autor traza una línea de acción representada por lo predeterminado y que no puede ser modificado. En el plano real, son las condiciones ambientales o ecológicas que el individuo no puede cambiar -por ejemplo, que el clima del Ártico frío-; mientras que en el ciberespacio se plantea la metáfora de la arquitectura o naturaleza. Para Lessig (1998), es el carácter constitutivo del código la ley de mayor trascendencia, ya que traza las mayores dimensiones de distancia entre el ciberespacio y el mundo real. Si bien en este último existen reglamentaciones impuestas por condiciones socio-económicas y culturales, fundadas en las luchas de poder entre los actores sociales -estatales, gubernamentales, privados, con y sin fines de lucro, organismos nacionales y supranacionales-, estas encuentran sus límites en la naturaleza del espacio de influencia o el escenario de acción.

Este último punto marca la gran diferencia con el ciberespacio, ya que la arquitectura o código que lo rige está configurado por programadores y diseñadores. El código, en palabras textuales del escritor de "Cultura Libre" es el conjunto de protocolos y reglas implementados o codificados -software y hardware- del ciberespacio mismo, los cuales determinan cómo interactúan o existen las personas en este espacio" (Lessig, 1998:2). Esta última regulación, a diferencia de las anteriores restricciones, no puede ser modificada pese a la voluntad de las partes, porque es inherente y constitutiva del espacio en red.

A partir de estas categorías, deben comprenderse los cuatro principios como factores correlacionados para englobar las acciones en red y cómo actúan en el plano regulatorio, ya que ninguno de ellos por si sólo puede representar el efecto de los cuatro juntos.

En este contexto, es pertinente conceptualizar el marco de situación del escenario donde se mueven estos nuevos actores, a fin de evitar confusiones metodológicas y establecer criterios de acción para los estudios de la comunicación.

$\mathrm{Si}$ bien los dispositivos tecnológicos crean las condiciones de posibilidad para la producción propia por parte de los usuarios, hay aspectos centrales del propio funcionamiento de la red que impiden la participación plena, así como las acciones sociopolíticas que impiden el acceso total de la población a los servicios de Internet.

Tal como afirma Tomás Maldonado (1998), hay dos grandes grupos que inciden en lo que atañe a las tecnologías: aquellas motivadas por los intereses económicos y por otro lado, los que le imprimen un carácter democratizador a los dispositivos de comunicación e información. Los primeros están atravesados por una actitud receptiva en cuanto a la suerte de las instituciones democráticas, mientras que los segundos reconocen dos aspectos que proceden en detrimento de la república electrónica.

Por un lado, el control normativo de los gobiernos sobre las redes y por el otro, los monopolios económicos. Desde estos argumentos, se infiere la tensión existente entre las dos corrientes teóricas que atraviesan el uso de la red, y que afectan la producción por parte de los usuarios, ya sea de contenidos audiovisuales o de información o interacciones sociales.

No obstante, es importante reconocer que en la arquitectura del ciberespacio se generan hibridaciones entre los medios, la cultura, el arte, herramientas multimedia o la realidad virtual que hacen posible un tipo de inteligencia colectiva, representativa de los tiempos actuales (Teniente, 2004:4).

En este punto, es trascendente comprender que el uso del lenguaje no responde a un proceso estático. La interacción entre seres humanos y máquinas se ha desarrollado como un fenómeno colectivo, orgánico, una mutación en las formas de apropiación y una propagación masiva de nuevos códigos (Ibídem).

\section{Consideraciones parciales}

El término prosumidor implica considerar el carácter transversal del usuario en la industria audiovisual, rol que, pese a los alcances, se distancia de lo denominado productor. Las acciones realizadas en la actualidad por el individuo o colectivo deben ser analizadas desde el plano de las interacciones y desde las propuestas de los productores, a fin de poder 
identificar la relación entre televisión y redes, así como la retroacción entre estos últimos y el receptor.

Si ahondamos en la conformación de la red, es posible identificar que su genealogía depende de la producción continua de los usuarios, desde contenidos gráficos o audiovisuales hasta todas las interacciones cotidianas que realizan en sus casillas de correos, portales digitales, redes sociales y plataformas colaborativas.

El desafío en la actualidad es encontrar un término que contemple la participación y las prácticas en red no desde el plano de la producción y el consumo, sino que se consideren los contenidos como bienes comunes y sea posible su copia y reproducción abierta y libre, sin condicionamientos de la industria, con el objetivo de impulsar y fortalecer políticas públicas orientadas a los derechos de acceso y participación de las TIC y mecanismos democráticos de regulación, universales y comunitarios. Desde esta perspectiva, es posible reivindicar difusiones descentralizadas, socialización de la información veraz, pluralismo cultural, redistribución del conocimiento al conjunto de los ciudadanos y división igualitaria de los beneficios tecno-científicos entre países, sociedades y pueblos (De Moraes, 2010:74-75)

En consecuencia, implica retomar el carácter colaborativo y participativo de la red, con el objetivo de no depender de los términos industriales para definir las prácticas sociales. Desde esta premisa, el desafío es pensar todo lo circulante en Internet como bienes comunes a todos, elemento que es útil para pensar en una política pública que permita el acceso y la participación real de todos los ciudadanos.

\section{Notas}

1.-Una sola función. Son los denominados medios tradicionales -radio y televisión- por ser dispositivos con una función específica: escuchar en el caso de la primera, visionado en el caso de la segunda.

2.-Pantallas cuya función oscila entre mirar televisión, reproducir películas, escribir y leer textos, dibujar, calcular, registrar y editar fotografías y vídeos, jugar o estudiar y socializar con otras personas -conocidas o no-, entre otras. La concepción multifunción contempla, además, la inclusión en estas pantallas de herramientas que compiten con otros medios, como el teléfono en el caso del programa Skype.

3.-Ariel Vercelli (2009) desarrolla el concepto de coconstrucción para referir a los cambios que impulsados por la articulación de los diversos actores, enfocado en la perspectiva de Derecho de Autor. Así, el término enfoca también a transformación de dispositivos móviles como consecuencia de las nuevas prácticas de los usuarios, donde tanto las tecnologías como los actores convergen en un proceso de co-construcción continuo.

4.- El concepto tiene por significado la analogía de estar en todas partes al mismo tiempo. Su relación con las TIC se establece por la posibilidad que ellas habilitan, a partir de las conexiones, de estar en diferentes lugares al mismo tiempo. Así, la conexión entre de lo real y lo virtual, la disponibilidad de la información a cualquier hora, desde cualquier lugar y con una variedad de dispositivos tecnológicos, modifican la forma de acceder a la información y al conocimiento (Islas, 2008).

5.- La GSMA representa los intereses de los operadores móviles en el mercado global. Se encuentra constituido por más de 220 países y reúne cerca de 800 operadores móviles del ámbito mundial con más de 250 compañías del ecosistema móvil. Incluye fabricantes de teléfonos, empresas de software, proveedores de equipos y compañías de Internet. Los datos fueron extraídos del informe anual de 2013, realizado por el Observatorio Móvil de América Latina. Disponible en http://www.gsmamobileeconomylatinamerica.com/SPA _LatAmME_v6_WEB_FINAL.pdf

6.-El concepto info-comunicación es un término que permite el estudio del mercado de las telecomunicaciones y los medios de comunicación. Refiere a la industrialización creciente de la información, de la cultura y de los intercambios sociales e incluye a las Industrias Culturales (prensa, libros, cine, televisión abierta y de pago, radio, fonogramas) y a las telecomunicaciones $\mathrm{e}$ Internet. Cfr: Mastrini, G.; Becerra, M. (2005). Estructura y dimensión de las industrias comunicacionales en América Latina. En Revista Palabra Clave, $\mathrm{N}^{\circ} 12$, Colombia. Disponible en http://www.redalyc.org/pdf/649/64901201.pdf

7.- Es necesario diferenciar los conceptos de "interacción" y "participación". Mientras que el primero hace referencia en las formas en las que se han diseñado las nuevas 
tecnologías para responder mejor a la noción del consumidor; el segundo se encuentra condicionado por los protocolos culturales y sociales. Por ejemplo, la cantidad de conversación posible en un cine se determina más por el grado de tolerancia entre los espectadores presentes en la sala que de alguno de los trabajadores del cine. La participación es más abierta, se encuentra menos controlada por los productores y más por los consumidores mediático (Jenkins, 2006:138-139).

8.-Cfr Becerra, M.; Hernández, P.; Postolski, G. (2003). La concentración de las industrias culturales. En Industrias Culturales: mercado y políticas públicas en Argentina, Buenos Aires: CICCUS.

9.-Twitter y Nielsen realizaron fusión a mediados de 2012 a fin de medir a la "audiencia social", es decir, las interacciones de los usuarios al momento de emitirse en vivo un programa de televisión. Cfr Twitter y Nielsen crean una herramienta para medir la participación de las audiencias de televisión. Recuperado el 23 de septiembre de

http: / /www.marketingdirecto.com/actualidad/medios/t witter-y-nielsen-crean-una-herramienta-para-medir-laparticipacion-de-las-audiencias-de-tv/ .

10.- Mediación tecnológica: la televisión, al ser un medio electrónico, produce su propia mediación y utiliza recursos para imponerla sobre su audiencia. El proceso de mediación de la televisión se origina en la elección de los formatos y géneros. Como parte de la particularidad del medio, es a través de la imagen que refuerza los altos grados de verosimilitud y representación que posee como medio electrónico audiovisual.

Cfr: Orozco Gómez, G. (2012). Televisión y producción de interacciones comunicativas. En Nueva época, $\mathrm{N}^{\circ} 18$, pp: 39-54.

11.-Con el objetivo de aprender de los tipos de interacción entre la producción y audiencia, el observatorio consensúa matrices de análisis de clasificación común. La clasificación fue orientada por el protocolo metodológico Obitel y esta constituida por las categorías: Interactiva; Interactiva en tiempo real; Visionado, Visionado interactivo; visionado interactivo en red y Visionado Transmediático. (OBITEL, 2012:63-64).
12.-Se denominan Contenidos Generados por el Usuario $(\mathrm{CGU})$. La sigla proviene del inglés "User Generated Content (UGC)"; y refiere a contenidos que el usuario puede alterar, editar, o a los que puede contribuir, tales como foros de discusión, blogs, wikis, sitios de relaciones, redes sociales, fotos, videos, comentarios, colaboraciones, intercambios de fotos o audio.

13.-Cfr: Williams, R. (1973). Televisión: Tecnología y Forma Cultural, Buenos Aires: Paidós.

14.-Entrevista a Manuel Castells. La socialidad de hoy está en Internet. Recuperado el 23 agosto de 2013 de http: / / www.revistaenie.clarin.com/ideas/Manuel-

Castells-sociabilidad-real-hoy-Internet_0_967703232.htm

15.-Actor que emerge gracias a la convergencia (Zallo, 2009). Uno de los ejemplos más representativos es el de Netflix, que facilita los contenidos de las grandes productoras a los usuarios.

Cfr: Ojer, T.; Capapé, E. (2012). Nuevos modelos de negocio en la distribución de contenidos audiovisuales: el caso de Netflix. En Revista Comunicación, Nº10, Vol.1, pp: 187-200.

16.- Cfr: McChesney, R. (2002). Economía política de los medios y las industrias de la información en un mundo globalizado. En José Vidal Beneyto, La ventana global: ciberespacio, esfera pública mundial y universo mediático, Barcelona: Editorial Taurus, pp: 233-248 y Cfr Mastrini, G.; Becerra, M. (2005). Estructura y dimensión de las industrias info-comunicacionales en América Latina. En Palabra Clave, $\mathrm{N}^{\mathrm{o}} 12$, junio, pp: 10-28.

17.-Con éxitos efímeros, algunas producciones de los usuarios trascienden el mercado. Sin analizar el fenómeno de Justin Bieber -que nació en Internet- encontramos los casos de vídeos que trascienden la escena local, como los realizados por los fanáticos de The Walking Dead, por mencionar ejemplos actuales.

Cfr Jenkins, H. (2006). La cultura de la convergencia de los medios de comunicación, España: Paidós

18.- Cfr Unión Internacional de Telecomunicaciones (2013). Medición de la Sociedad de la Información, Ginebra, Suiza: Place des Nations, CH-1211.

\section{Referencias}

- Amado, A. (2013). Televidente-ciudadano busca televisión televisiva. En Rincón, O. (Editor) Zapping TV: el paisaje de la tele latina. Recuperado el 23 de noviembre de 2013 de http://library.fes.de/pdf-files/bueros/lacomunicacion/10264.pdf. 
Casajús, L.; Guerrero, M.V. (2014). Los jóvenes prosumidores en la Radio Universitaria 2.0. Un perfil en construcción, EDMEMIC 3 (1), pp: 87-111. Recuperado de http://www.edmetic.es/Documentos/Vol3Num12014/Vol3Num1-5.pdf el 12 de julio de 2014.

- De Moraes, D. (2010). Cultura tecnológica, innovación y mercantilización. En De Moraes, D. (comp.). Mutaciones de lo invisible. Comunicación y procesos culturales en la era digital, Argentina: Paidós; pp: 45-81

- Becerra, M. (2010). Mutaciones en la superficie y cambios estructurales. América Latina en Parnaso informacional. En De Moraes, D. (comp.), Mutaciones de lo invisible. Comunicación y procesos culturales en la era digital, Argentina: Paidós; pp: 81-113

- Fernández, P. (2013). Las audiencias en la era digital: interacción y participación en un sistema convergente. En Revista Question, Vol. $1 \mathrm{~N}^{\circ} 40$. Disponible en http: / / perio.unlp.edu.ar/ojs/index.php/question/article /view/1957. Última consulta: 20 de diciembre de 2013.

- García Fanlo, L. (2012). Twitter y la rebelión de los ciberfans de Gran Hermano. En La política de los internautas, Buenos Aires: La Crujía, pp:75-102

- Igarza, R. (2008). Nuevos medios: estrategias de convergencia, Argentina: La Crujía.

- Igarza, R. (2009) Burbujas de Ocio. Nuevas formas de consumo cultural, Argentina: La Crujía.

- Islas, O. (2008) El prosumidor. El actor comunicativo de la sociedad de la ubicuidad. Recuperado el 23 de noviembre de 2013 de http: / / octavioislas.files.wordpress.com/2009/09/octavi oislas_2008_coneicc.pdf.

- Islas, O. (2014). La sociedad de la ubicuidad, los prosumidores y un modelo de comunicación para comprender la complejidad de las comunicaciones digitales. En Revista Latinoamericana de Ciencias de la Comunicación, $\mathrm{N}^{\mathrm{o}}$ 7, julio-diciembre, Brasil, pp. 68-77. Recuperado de http: / / www .razonypalabra.org.mx/N/n65/varia/oislas. html el 12 de julio de 2014.

- Jenkins, H (2008) La cultura de la convergencia de los medios de comunicación, España: Paidós

- Jenkins, H. (2009) Fans, blogueros y videojuegos. La cultura de la colaboración, España: Paidós

- Levis, D (2011) El mundo en sus pantallas. En Indicadores culturales 2010, Argentina: Instituto de políticas culturales Patricio Lóizaga de la Universidad Nacional Tres de Febrero, pp: 138-144

- Lessig, L. (1998) Las leyes del ciberespacio. Recuperado el 23 de noviembre de 2013 de http://www.uned.es/ntedu/espanol/master/segundo/m odulos/audiencias-y-nuevos-medios/ciberesp.htm.

- Maldonado, T(1998) Crítica de la razón informática, España: Paidós

- Martín Barbero, J. (1987) De los medios a las mediaciones, México. Editorial Gilli

- Martín Barbero, J. (2010) Convergencia Digital y diversidad Cultural. En De Moraes, D. (comp.), Mutaciones de lo invisible. Comunicación y procesos culturales en la era digital, Argentina: Paidós, pp: 137-165

- Negroponte, N. (1999) Ser Digital, Argentina: Atlántida

- McChesney, R. (2002), Economía política de los medios y las industrias de la información en un mundo globalizado. En Vidal Beneyto, José (director), La ventana global, España: Taurus, pp: 233-247.

- Mcluhan, M.; Barrington N. (1972). Take Today: The Executive as Dropout, Canadá: Harcourt Brace Jovanovich.

- McLuhan, M (1997). El medio es el mensaje, España: Paidós

- Observatorio Iberoamericano de la Ficción Televisiva (OBITEL) (2011). Calidad de la ficción televisiva y participación transmediática de las audiencias, Brasil: Editorial Globo. Disponible en http: / /blogdoobitel.files.wordpress.com/2011/04/espac 3b1ol.pdf Última consulta: 12 de julio de 2014.

- Observatorio Iberoamericano de la Ficción Televisiva (2012). Transnacionalización de la ficción televisiva en los países iberoamericanos, Brasil: Merdional Ltda. Disponible en: http: / / blogdoobitel.files.wordpress.com/2011/04/obite 12012espanhol-120912131100-phpapp02.pdf Última consulta: 12 de julio de 2014 .

- Orozco Gómez, G. (1994a). Recepción televisiva y mediaciones. La construcción de estrategias por la audiencia. En Cuadernos de Comunicación y Prácticas Sociales, $\mathrm{N}^{\circ} 6$, México: UIA.

- Orozco Gómez, G. (1994b). Televisión y producción de significados (Tres ensayos), México: DECS/Universidad de Guadalajara

- Orozco Gómez, G. (1997). Medios, audiencias y mediaciones, España: Comunicar $\mathrm{N}^{\circ} 8$

- Orozco Gómez, G. (2001) Audiencias, televisión y educación: una de-construcción pedagógica de la televidencia y sus mediaciones, México: Revista Iberoamericana de Educación, No27 pp: 155-175. 
- Orozco Gómez, G. (2009). Entre pantallas: nuevos roles comunicativos de sus audiencias-usuarios. En Aguilar, M; Nivón, E; Portal, M; Wincour, R (Coord.), Pensar lo contemporáneo: de la cultura situada a la convergencia tecnológica, España/México: Editorial Anthropos y Universidad Autónoma Metropolitana

- Orozco Gómez, G. (2011)"Estar” como audiencia y "Ser" audiencia en el siglo XXI. En "Indicadores culturales 2010”, Argentina: Instituto de políticas culturales Patricio Lóizaga de la Universidad Nacional Tres de Febrero. pp: 87-97

- Orozco Gómez, G. (2012). Televisión y producción de interacciones comunicativas, México: Nueva época, $\mathrm{N}^{\circ} 18$, pp:39-54.

- Rincón, O. (Editor). Zapping TV: el paisaje de la tele latina, México: Friedrich Ebert Stiftung:FES COMUNICACIÓN. Recuperado de http://library.fes.de/pdffiles/bueros/la-comunicacion/10264.pdf el 12 de julio de 2013.

- $\quad$ Rheingold, H. (1996). La comunidad virtual: una comunidad sin fronteras, Barcelona: Gedisa.

- $\quad$ Scolari, C. (2009). Hipermediaciones. Elementos para una Teoría de la Comunicación Digital Interactiva, España: Gedisa.

- Sued, G. (2010). Pensando en Facebook: una aproximación colectiva por dimensiones. En Piscitelli, A.; Adaime, I.; Binder, I. (comps), El proyecto Facebook y la posuniversidad: sistemas operativos sociales y entornos abiertos de aprendizaje, España: Ariel, fundación Telefónica. pp: 51-69
- Teniente, E.D.A (2004). Jóvenes "prosumidores" de Internet en los dispositivos móviles. Recuperado de http: / / entretextos.leon.uia.mx/num/15/PDF/ENT157.pdf el 12 de julio de 2014.

- Vercelli, A. (2009) Repensando los bienes intelectuales comunes: análisis socio-técnico sobre el proceso de co-construcción entre las regulaciones de derecho de autor y derecho a copia y las tecnologías digitales para su gestión. Recuperado el 23 de noviembre de 2013 de http: / / www.arielvercelli.org/rlbic.pdf.

- Vasallo de Lopes, M.I. (2012). Un estudio de caso de recepción transmediática: comunidades de fans en Facebook y temas sociales de la telenovela brasileña Passione. En Carlón, M.; Scolari, C. Colaborarte. Medios y artes en la era de la producción colaborativa, Buenos Aires: La Crujía.

- Villarreal, G (2010). Lukers del mundo 2.0 ¡Unios! En Medios y enteros N6. Recuperado el 17 de marzo de 2012 de www.newsmatic.epol.com.ar $/$ index.php?pub_id $=549 \& \operatorname{sid}=5645 \& \mathrm{raid}=640$ $63 \&$ reid $=6 \&$ NombreSeccion $=$ Portada $\&$ Accion $=$ VerArtic ulo

- Zallo, R. (2007). La economía de la cultura (y de la comunicación) como objeto de estudio. Recuperado el 23 de noviembre de 2013 de http: / / www.ehu.es/zer/hemeroteca/pdfs/zer22-10zallo.pdf .

\section{Forma de Citación}

FERNÁNDEZ, Paola: Consumos culturales en América Latina y la emergencia del prosumidor: Un recorrido conceptual desde la Sociedad de la Información. Revista Communication Papers, N $\mathrm{N}^{\mathrm{0}}$, páginas 87 a 100. Departamento de Filología y Comunicación de la Universidad de Girona. Recuperado el _ de de 2 de: http://www.communicationpapers.es 